\title{
GPU-Based Segmented-Beat Modulation Method for Denoising Athlete Electrocardiograms During Training
}

\author{
Amnah Nasim, Edoardo Della Santa, Damiano Tanchi, Agnese Sbrollini, Ilaria Marcantoni, Micaela \\ Morettini, Laura Burattini \\ Università Politecnica delle Marche, Ancona, Italy
}

\begin{abstract}
Sport-related sudden cardiac death (SRSCD), defined as "death occurring during sport or within one hour of cessation of training", is the leading cause of death in athletes. SRSCD occurs in the presence of underlying cardiovascular diseases, some of which may be identified by processing electrocardiographic recordings acquired during training (TECGs). A fast and accurate processing of TECGs during or immediately after training is challenging since TECGs are typically highly corrupted by noise and interferences, which may jeopardize their interpretation and identification of abnormal morphologies. The present study evaluated the ability of GPU-based Segmented-Beat Modulation Method (GPU$S B M M)$ to provide a noise-free estimation of TECGs, and to improve the algorithm by GPU acceleration to make it compatible with modern hardware. In this research, 196 to-10 min TECGs (sampling frequency: $256 \mathrm{~Hz}$ ), acquired from 8 subjects while performing 4 different exercise tasks (walk, run, low-resistance bike and high-resistance bike), were analyzed. Results indicate that GPU-SBMM application yielded a significant increase of $\operatorname{SNR}(d B)$ (from $1 \pm 5 d B$ to $19 \pm 5 d B ; p<10^{-12}$ ), also when stratifying by exercise tasks. Additionally, a considerable average speedup of 7.67x is achieved using NVIDIA GeForce 740M GPU processor.
\end{abstract}

\section{Introduction}

Sport-related sudden cardiac death (SRSCD), defined as "death occurring during sport or within one hour of cessation of training", is the leading cause of death in athletes. A recent estimate suggests that 1 in 40,000 to 1 in 80,000 athletes per year [1] suffer from a sudden cardiac event resulting in either death or a non-fatal arrhythmia. This occurrence is still 3-4 times more often for athletes as compared to non-athletes [2]. This increase in the events of SRSCD in athletes in recent past poses a serious concern for the physicians and trainers responsible for the health and well-being of players [3]. Davide Astori (1987-2018) aged 31, Neil Fingleton (1980-2017) aged 36, Fabricio Paulino de Melo (1990-2017) aged 26 and Bernardo Ribeiro (1989-2016) aged 26 are a few examples of professional athletes who lost their lives due to sudden cardiovascular arrest lately and the incidents left families and fans completely heartbroken and full of questions.

SRSCD occurs in the presence of underlying cardiovascular diseases, some of which may be identified by processing electrocardiographic recordings (ECG) acquired during training (TECG), eventually using wearable devices [4]. According to data, the probability of the ECG to detect underlying cardiovascular abnormalities that could place the athlete at risk of SRSCD is superior to that of physical examination and history [5,6]. However, TECGs in the acquired raw form are affected by several additional noise factors such as electrode motion artefacts, muscular artefacts emanating from intense and regular physical activities like running, walking, gym training etc. that can make the signal of interest clinically useless [7]. Additionally, the transmission of large amount of TECG data consumes a great deal of energy and reduces the battery life of both the wearable device and the processing platform [8]. To extend battery life, high-end processing platforms are required to have in-time identification and alert generation for prevention of SRSCD. The Segmented-Beat Modulation Method (SBMM) [9,10] provides a template-based ECG filter with physiological heart-rate and morphological variability. It has previously been tested in applications relative to abdominal fetal ECG [11] and electromyography filtering from ECG corruption [12], but never to filter TECG. The highly parallelizable and independent nature of data processing with SBMM makes it suitable to be implemented on high performance computing programming structures and platforms. A CPUbased approach uses single instruction single data (SISD) programming structure executing one program statement operating on one data stream at a time. Whereas, GPU architecture is characterized by single instruction multiple data (SIMD) feature [13], which allows operations on multiple data points simultaneously. GPU provides a highperformance hardware platform for faster simulations. Thus, in this work, GPU-based SBMM (GPU-SBMM) is 
proposed as performance improved version of the previously proposed CPU-based SBMM (SBMM) for the denoising of TECG data.

\section{Materials and Methods}

\subsection{Data}

Single-channel TECG recordings (sampling frequency: $256 \mathrm{~Hz}$ ) from 8 subjects (3 male, 5 female), aged 22-32 years (mean $26.5 \pm 5.5$ years) were used. Measurements were taken using an Actiwave (CamNtech, Cambridge, UK) ECG recorder placed on the chest while participants were asked to perform one or more exercise tasks (walk, run, low-resistance bike and high-resistance bike) as summarized in Table 1. Data were taken from the "Wrists PPG During Exercise" database by Physionet [14,15]

All TECG recordings were band-pass filtered through a bidirectional $3^{\text {rd }}$-order Butterworth filter with cut-off frequencies of $0.5 \mathrm{~Hz}$ and $40 \mathrm{~Hz}$. After that, the baseline was removed. Baseline was computed as a cubic spline interpolation of fiducial points, placed $90 \mathrm{~ms}$ before Rpeaks. Annotations of the reference TECG R-peak positions were provided in the Physionet database. The mean HR (heart rate) and HRV (HR variability measured as RR-interval standard deviation) was $104 \pm 25 \mathrm{bpm}$ and $54 \pm 26 \mathrm{~ms}$, respectively.

\subsection{GPU-based Segmented-Beat Modulation Method (GPU-SBMM)}

GPU-SBMM was implemented in MATLAB using Parallel Computing Toolbox built-in functions. GPU-
SBMM takes as input the noisy TECG signal, the R-peak positions vector and some initial settings (TECG sampling frequency and gain). To enter GPU-SBMM processing, data have been copied from the CPU to the GPU device through the 'gpuArray()' function. Each cardiac cycle, which in GPU-SBMM can be processed independently from the others, was segmented into QRS and TUP segments.

After the segmentation of cardiac cycles into QRS and TUP segments, a resampling of TUP segments is performed to match the TUP length of median cardiac cycle (mCC) of all beats in a single recording. A peak correction is applied to identify and correct falsely detected peaks.

Finally, GPU-SBMM performs a filtering procedure that reconstructs a clean TECG signal from the one corrupted by noise recording. All the for-loops for segmentation, modulation, correlation optimization and reconstruction steps are implemented as vectorized cell array statements using 'cellarray()' function. The computed clean TECG from the GPU processor is then sent back to CPU using 'gather()' function. The distribution of workload for GPU-SBMM is shown in Figure 1.

\subsection{Computing Platform}

All GPU experiments were performed on NVIDIA GeForce GT 740M processing machine with compute capability 3.5 based on the Kepler architecture and 64GB GPU memory with bandwidth of $173 \mathrm{~GB} / \mathrm{s}$.

All CPU experiments were performed on Intel® Core i5 with 2 cores and an operating frequency of $1.8 \mathrm{GHz}$.

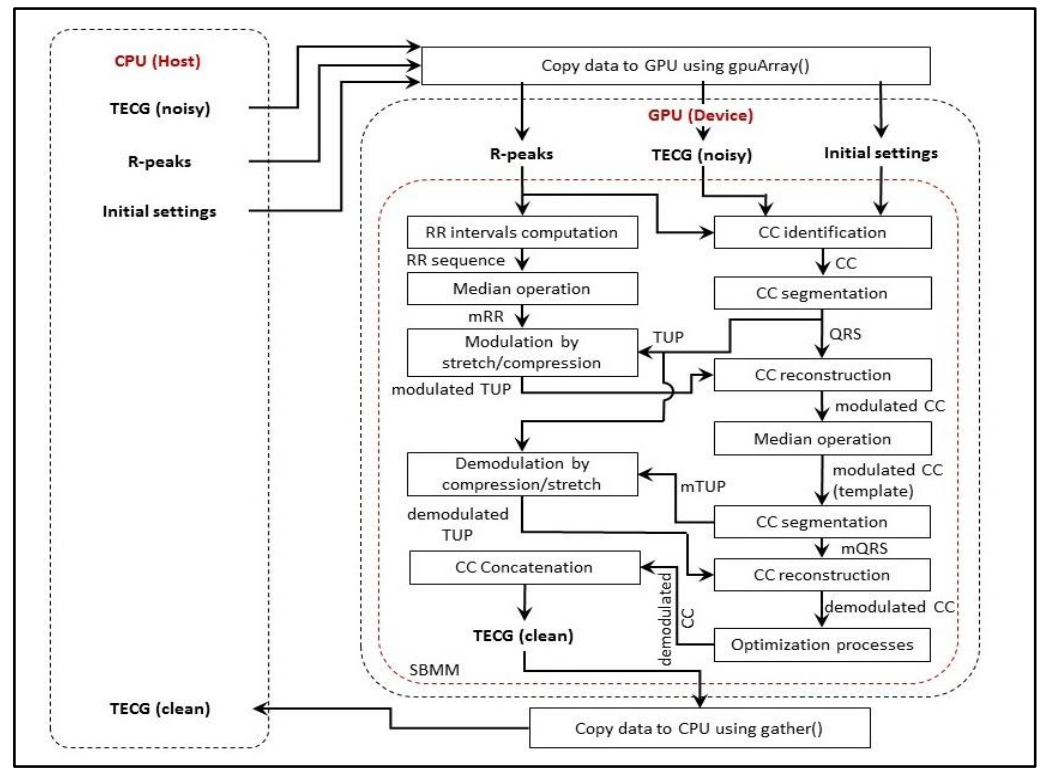

Figure 1. Distribution of workload for GPU-based Segmented-Beat Modulation Method. 


\subsection{Assessment of signal quality improvement}

To quantify the reliability of the GPU-SBMM in reducing the level of noise affecting the TECG signals, the signal-to-noise ratio (SNR) expressed in decibel (dB) was computed before (noisy) and after (clean) GPU-SBMM processing according to equation (1):

$$
\operatorname{SNR}(d B)=10 \log _{10}\left\lfloor\left(\frac{A_{\text {Signal }}}{A_{\text {Noise }}}\right)^{2}\right\rfloor,
$$

where $\mathrm{A}_{\text {Signal }}(\mathrm{mV})$ is the signal amplitude and $\mathrm{A}_{\text {Noise }}(\mathrm{mV})$ is the noise amplitude. Amplitudes of TECGs being close to deterministic (pseudo-periodic) signals, were computed as mean of the maximum-minus-minimum values over the beats. Whereas, amplitudes of noise signals being close to Gaussian stochastic signals were computed as 4 times standard deviation [12]. All amplitude values were computed over the entire length of the study records.

\subsection{Assessment of speed of execution improvement}

For each TECG signal, GPU-SBMM runtime $\left(t_{\mathrm{GPU}}\right.$ SBмм, s) was computed. Moreover, each TECG signal was processed with the sequential SBMM implementation [10] and the SBMM runtime $\left(\mathrm{t}_{\text {SBMM }}, \mathrm{s}\right)$ was computed. To show the effect on performance after GPU resource enhancement, speedup factor (Speedup(x)), defined by Amdahl's law [16] as an improvement in the speed of execution of a task on two computing architectures with different resources, was computed for each TECG signal using equation (2):

$$
\text { Speedup }(x)=\frac{t_{\mathrm{SBMM}}}{t_{\mathrm{GPU}-\mathrm{SBMM}}} .
$$

\subsection{Statistical analysis}

Differences between SNR before and after GPUSBMM processing have been evaluated by using a paired Student's t-test. Values are reported as mean \pm standard deviation (SD), unless otherwise specified. The two-sided significance level was set at 5\% ( $<<0.05)$.

\section{Results}

An example of the TECG signal recorded during exercise before and after GPU-SBMM processing is shown in Figure 2.

SNR values for each activity before and after GPUSBMM processing are given in Table 2. Mean SNR values
Table 1. Duration of TECG signals recorded for different subjects and activities.

\begin{tabular}{ccccc}
\hline Subject ID & Walk & Run & LRB & HRB \\
\hline 1 & $9: 48$ & - & $9: 39$ & $9: 18$ \\
2 & $6: 39$ & - & $5: 41$ & $6: 54$ \\
3 & $4: 47$ & $5: 07$ & $4: 54$ & $4: 41$ \\
4 & - & $4: 52$ & - & - \\
5 & - & $5: 08$ & $4: 40$ & - \\
6 & $5: 36$ & $5: 02$ & $4: 40$ & - \\
7 & $6: 42$ & $4: 47$ & - & - \\
8 & $3: 40$ & - & - & - \\
\hline \multicolumn{7}{l}{ LRB-Low-Resistance Bike } & & \\
HRB-High-Resistance Bike &
\end{tabular}

computed for all noisy TECG and clean TECG signals were $1 \pm 5 \mathrm{~dB}$ and $19 \pm 5 \mathrm{~dB}$, respectively; a significant increase $\left(\mathrm{p}<10^{-12}\right)$ in SNR was observed after GPU-SBMM processing. Stratifying for each exercise task, a significant increase in SNR was also found (Table 2).

The calculated SBMM and GPU-SBMM runtimes are shown in Figure 3. The GPU implementation reduces the runtime of the noise reduction algorithm from $4.82 \mathrm{~s}$ to $0.63 \mathrm{~s}$. Hence, the speedup achieved is 7.67 times higher than that of the CPU-based SBMM implementation.

\section{Discussion}

The present study proposed GPU-SBMM as performance improved version of SBMM for the denoising of TECGs. In the beat-wise segmentation, modulation and signal reconstruction steps characterizing GPU-SBMM, each beat can be processed simultaneously, because the processing of any individual beat is independent of the other, which is ideal for GPU processing. Because of the advantage that MATLAB is an interpreted language, inefficient for-loops in SBMM implementation have been replaced by vectorized statements to further optimize runtimes.

Improvement in SNR showed that GPU-SBMM is an effective and fast method for removal of exercise-induced noise factors in the TECG data. Comparative testing showed that the average speedup achieved for the four training activities is 7.67x. Offloading the heavy part of computation to the GPU processor which can either be a mobile platform or a desktop, the performance of the algorithm is increased. This allows an effective processing and analysis of TECG signals measured from wearable sensors through innovative software applications for the prevention of SRSCD [4].

For future recommendation, full benefits of parallelism for GPU-SBMM might be seen when used with big data (larger datasets or online ECG data processing). Moreover, a high denoising efficiency with TECGs makes GPUSBMM a useful method for a wearable ECG sensing apparatus/setting. 
Table 2. Comparative evaluation of SNR by activity.

\begin{tabular}{lccc}
\hline $\begin{array}{c}\text { Exercise } \\
\text { tasks }\end{array}$ & $\begin{array}{c}\text { No. of } \\
\text { TECG } \\
\text { s }\end{array}$ & $\begin{array}{c}\text { Before } \\
\text { SNR } \\
(\mathrm{dB})\end{array}$ & $\begin{array}{l}\text { After } \\
\text { SNR } \\
(\mathrm{dB})\end{array}$ \\
\hline Walk & 6 & $-2 \pm 3$ & $18 \pm 5^{*}$ \\
Run & 5 & $1 \pm 7$ & $20 \pm 7^{*}$ \\
Low Resistance Bike & 5 & $3 \pm 5$ & $20 \pm 5^{*}$ \\
High Resistance Bike & 3 & $3 \pm 6$ & $20 \pm 6^{*}$ \\
\hline
\end{tabular}

$* \mathrm{p}<0.05$

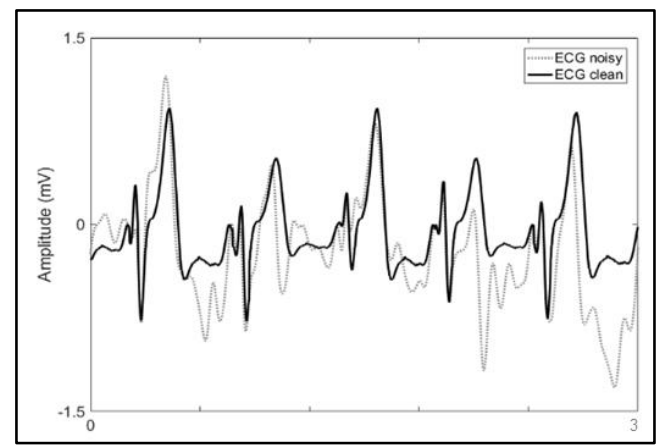

Figure 2. TECG plotted for the exercise task High Resistance Bike for duration of 3 seconds.

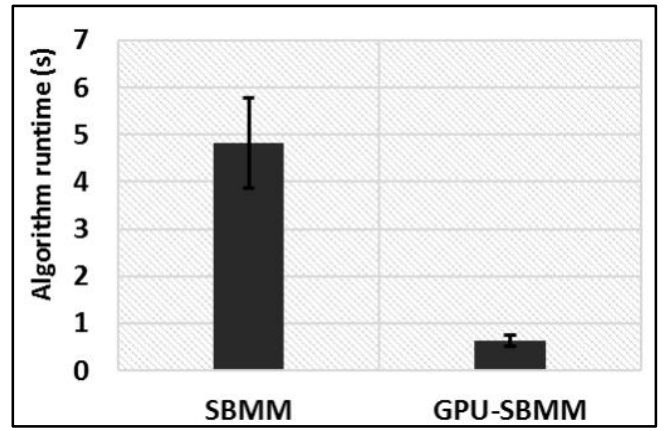

Figure 3. Runtime for CPU-based SBMM and GPU-based SBMM on NVIDIA GeForce GT 740 processor.

\section{Conclusion}

GPU-SBMM is an efficient and accurate algorithm for filtering TECG, typically affected by high level of noise. Thus, it represents a potentially useful tool to fight SRSCD.

\section{Acknowledgements}

This work was partially funded by Fondazione Cariverona, Italy.

\section{References}

[1] K. G. Harmon, J. A. Drezner, M. G. Wilson, and S. Sharma, "Incidence of sudden cardiac death in athletes: a state-of-theart review," Heart, vol. 100, no. 16, pp. 1227-1234, Aug. 2014.
[2] X. Jouven, W. Bougouin, K. Narayanan, and E. Marijon, "Sudden cardiac death and sports," J. Eur. Heart, vol. 38, no. 4, pp. 232-234, Jan. 2017.

[3] J. A. Drezner, S. Sharma, and M. G. Wilson, "Sports cardiology: preventing sudden cardiac death," Br. J. Sports Med., vol. 48, no. 15, pp. 1133, Aug. 2014.

[4] A. Agostinelli et al., "CaRiSMA 1.0: Cardiac risk selfmonitoring assessment," Open Sports Sci. J., vol. 10, no. 1, pp. 179-190, Oct. 2017.

[5] M. S. Emery and R. J. Kovacs, "Sudden cardiac death in athletes," JACC Heart Fail., vol. 6, no. 1, pp. 30-40, Jan. 2018.

[6] K. G. Harmon, M. Zigman, and J. A. Drezner., "The effectiveness of screening history, physical exam, and ECG to detect potentially lethal cardiac disorders in athletes: a systematic review/meta-analysis," J. Electrocardiol., vol. 48, no. 3, pp. 329-338, Jun. 2015.

[7] S. Nagai, D. Anzai, and J. Wang, "Motion artefact removals for wearable ECG using stationary wavelet transform," Healthc. Technol. Lett., vol. 4, no. 4, pp. 138, Jun. 2017.

[8] S. Liu, A. Bustin, D. Burshka, and A. Menini, "GPU implementation of Levenberg-Marquardt optimization for T1 mapping," Comput. Cardiol., vol. 44, 2017.

[9] A. Agostinelli et al., "Segmented beat modulation method for electrocardiogram estimation from noisy recordings," Med. Eng. Phys., vol. 38, no. 6, pp. 560-568, Jun. 2016.

[10] A. Agostinelli, C. Giuliani, and L. Burattini, "Extracting a clean ECG from a noisy recording: a new method based on segmented-beat modulation," Comput. Cardiol., vol. 41, pp. 49-52, 2014.

[11] A. Agostinelli et al., "Noninvasive fetal electrocardiography part II: Segmented-Beat Modulation Method for signal denoising," Open Biomed. Eng. J., vol. 11, pp. 25-35, Mar. 2017.

[12] A. Sbrollini et al., "Evaluation of the low-frequency components in surface electromyography," Conf. Proc. IEEE Eng. Med. Biol. Soc. 2016, pp. 3622-3625, 2016.

[13] A. Nasim, A. Maqsood, and T. Saeed, "Multicore and GPU based pupillometry using parabolic and elliptic Hough Transform," Int. J. Mech. Eng. Rob. Res., vol. 6, no. 5, pp. 425-433, Sept. 2017.

[14] D. Jarchi, and A. J. Casson, "Description of a database containing wrist PPG signals recorded during physical exercise with both accelerometer and gyroscope measures of motion," Data, vol. 2, no. 1, pp. 1-13, Dec. 2016.

[15] A. L. Goldberger et al., "PhysioBank, PhysioToolkit, and PhysioNet: components of a new research resource for complex physiologic signals," Circulation, vol. 101, no. 23, pp. e215-e220, Jun. 2000.

[16] M. Amdahl, "Validity of the single processor approach to achieving Large-Scale computing capabilities," Spring Joint Computer Conf., pp. 483-485, Apr. 1967.

Address for correspondence.

Laura Burattini, $\mathrm{PhD}$

Università Politecnica delle Marche

Department of Information Engineering (DII),

Via Brecce Bianche, 60131 Ancona, Italy

Email: 1.burattini@univpm.it 